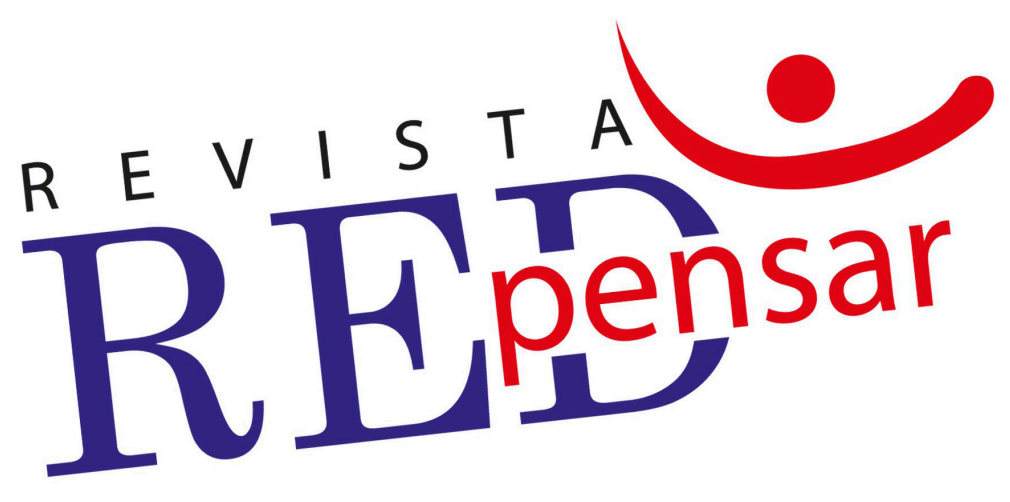

\title{
Biopedagogía: una clave para la supervivencia
} humana

Biopedagogy: a key to buman survival

Erika Badilla Sánchez - Viriam Mejias Padilla - Anny Tattiana Sancho Miranda

Revista REDpensar, volumen 9, número 1, Enero-Junio 2020 ISSNe: 2215-5384

Lanzando la RED 2

Recibido: 21 de abril de 2020 Aceptado: 08 de junio de 2020

DOI: 10.31906/redpensar.v9i1.201

\section{(ㄷ)(1) $\$(0)$}




\title{
Biopedagogía: \\ una clave para la supervivencia humana
}

\author{
Biopedagogy: a key to human survival
}

\author{
Erika Badilla Sánchez ${ }^{1}$ \\ Viriam Mejías Padilla ${ }^{2}$ \\ Anny Tattiana Sancho Miranda ${ }^{3}$
}

\section{Resumen}

El presente artículo reflexiona sobre una gran interrogante latente en la humanidad, ¿qué es la vida? Entre estas, han predominado las que responden al mecanicismo cartesiano y la reducen a un conjunto de mecanismos físicoquímicos y leyes universales. Pero, si bien esta perspectiva impulsó la investigación científica y el conocimiento del universo como nunca antes, es responsable de la destrucción del planeta Tierra. Algunas personas, guiadas por una visión holística del universo, entre científicas y pensadores adscritos a la perspectiva de la complejidad, llevaron adelante una importante dilucidación sobre el carácter esencial de la vida; gracias a sus aportes, la visión jerárquica de la naturaleza ha sido superada por una mirada de carácter rizomático, que, alimentada por nociones como autoorganización, autopoiesis y bioaprendizaje, constituyen el sustrato adecuado para que germine una propuesta de mediación pedagógica capaz de transformar completamente la sociedad y detener la destrucción de nuestra amada Gaia.

Palabras Clave: GAIA, HUMANIDAD, COMPLEJIDAD, HOLÍSTICA, BIOPEDAGOGÍA, COVID-19.

\section{Abstract}

This article reflects on a great latent question in humanity, what is life? Among these, those that respond to cartesian mechanism have predominated and reduce it to a set of physicochemical mechanisms and universal laws. But, while this perspective prompted scientific research and knowledge of the universe like never before, it is responsible for the destruction of planet Earth. Some people, guided by a holistic view of the universe, among scientists and thinkers attached to the perspective of complexity, carried out an important elucidation on the essential character of life; thanks to their contributions, the hierarchical vision of nature has been overcome by a rhizomatic look, which, fed by notions such as self-organization, autopoiesis and biolearning, constitute the adequate substrate for the germination of a pedagogical mediation proposal capable of completely transforming the society and stop the destruction of our beloved Gaia.

\footnotetext{
1. Costarricense. Estudiante del Doctorado en Educación con especialidad en Mediación Pedagógica. Magister en Salud Mental y Enfermería. Actualmente se desempeña como Enfermera especialista en Salud Mental en el Hospital San Juan de Dios, Caja Costarricense de Seguro Social. Además, imparte los cursos de Psicología infantil, Campo Clínico en Salud Mental y Comportamiento Organizacional en el Programa de Posgrado de Enfermería, de la Universidad Hispanoamericana. Correo electrónico: eka.badilla@gmail.com

2. Estudiante del Doctorado en Educación con especialidad en Mediación Pedagógica, Magister en Gerencia de Enfermería. Actualmente se desempeña como Directora de Enfermería del Hospital San Juan de Dios, Caja Costarricense de Seguro Social. Además, funge como Directora del Posgrado de Enfermería de la Universidad Hispanoamericana, programa en el cual imparte los cursos de Gestión de Servicios de Enfermería I y Supervisión y Evaluación del Desempeño. Correo electrónico: vmejias@,ccss.sa.cr

3. Estudiante del Doctorado en Educación con especialidad en Mediación Pedagógica, Magister en Enfermería Pediátrica con Énfasis en Neonatología, Magister en Gerencia de Enfermería. Actualmente se desempeña como Jefe de Unidad Servicio de Neonatología, Hospital San Juan de Dios, Caja Costarricense de Seguro Social. Además, imparte los cursos de Enfermería Clínica I y II de la Maestría de Enfermería Pediátrica de la Universidad Hispanoamericana. Correo electrónico: asanchom@,ccss.sa.cr
} 
Keywords: GAIA, HUMANITY, COMPLEXITY, HOLISTICS, BIOPEDAGOGY, COVID-19.

\section{Introducción}

A principios de los años sesenta, el científico y ambientalista James Lovelock (nacido, no por casualidad, en la Ciudad-Jardín de Letchwort ${ }^{\uparrow}$ inició la formulación de la hipótesis Gaia. Un modelo interpretativo del planeta Tierra, que según Lovelock (1983) la biosfera constituye un super organismo que se autorregula y auto transforma para asegurar la supervivencia de la vida.

En el actual contexto de crisis global por cambio climático y la pandemia por COVID-19, se ha renovado el interés por este modelo. Dentro de las tesis sobre el origen del virus SARS-CoV2, una de las más llamativas se deriva de la hipótesis Gaia, ya que Lovelock dejó muy en claro, la capacidad de autorregulación y autotransformación de la biosfera, lo cual, le permite adaptarse a fenómenos tan graves como la contaminación industrial, las guerras atómicas, químicas o biológicas, acudiendo a dientes y garras, en caso de ser necesario ${ }^{5}$ (Lovelock, 1983, p.87).

Si bien esta idea es rechazada por la mayoría de la comunidad científica internacional, la cual la califica como resultado de un intento por atribuirle voluntad al planeta Tierra, o una simple locura; desde el pensamiento complejo la propuesta de Lovelock ha llegado a alimentar la hipótesis de que patógenos como el SARS-CoV-2, son en realidad una consecuencia de los graves desequilibrios ambientales causados por el ser humano (O'callaghan, 2020), así como una respuesta de autodefensa por parte de la naturaleza.

Una de las principales impulsoras de la hipótesis Gaia fue la bióloga Lynn Margulis (1938-2011†), considerada una de las principales figuras contemporáneas en el campo de la evolución biológica. Quien incorporó la idea de que la biosfera constituye un único sistema viviente y una holarquía global.

Margulis recupera así una de las tesis fundamentales de Arthur Koestler (activista político y filósofo social), según la cual los sistemas (holarquías) puede ser entendidas como totalidades, en el tanto cada una de sus partes (holones ${ }^{\natural}$ ) no se caracteriza por su posición jerárquica, sino por constituir a su vez otra totalidad, constituida de otras partes y así sucesivamente (Margulis y Sagan, 2005).

Margulis y Sagan (2005) señalan que la evolución, más que descansar en la competencia entre las distintas formas de vida que germinan en la biosfera, ha sido posible por un principio básico de simbiosis: "la vida en la Tierra no es una jerarquía creada, sino una holarquía emergente surgida de la sinergia autoinducida de combinación, acoplamiento y recombinación” (Margulis y Sagan, 2005, p. 18).

Si bien se debe reconocer que la propuesta de Lovelock (1983) y Margulis y Sagan (2005) no tuvo el eco esperado en el mundo de la ciencia positivista, la cual sigue imponiendo la asimilación de la vida a un mero conjunto de reacciones químicas, en el campo de las ciencias humanas y sociales. La hipótesis Gaia se ha convertido en una poderosa metáfora filosófica que nos obliga a reflexionar sobre el lugar que ocupa el ser humano en el entramado de la vida.

\footnotetext{
4. La Ciudad Jardín de Letchworth fue ideada en 1898 por Ebenezer Howard, un joven periodista inglés que durante su vida en Nebraska (EEUU) estuvo en contacto Walt Whitman y Ralph Waldo Emerson, quienes influyeron en su pensamiento en la búsqueda de comunidades más aptas para el bienestar del ser humano en armonía con la naturaleza. Las ciudades jardín fueron pensadas por Howard como un medio para reconstruir la sociedad capitalista, convirtiéndola en una infinidad de sociedades cooperativas autónomas mediadas por entornos urbano-rurales donde el bien común y el contacto directo con la naturaleza fueran la norma (Blasco, 2016).

5. Tesis que cabe al menos valorar frente al desacuerdo científico en torno del origen de las pandemias que asolan la humanidad en el siglo XXI, entre ellas el COVID-19, atribuida por algunos al desequilibrio ambiental generado por el ser humano.

6. Término acuñado por Arthur Koestler, para referir a aquellos sistemas que son al mismo tiempo todo y parte, en tanto forman parte de otros sistemas mayores y cada una de sus partes constituye a su vez un sistema (Koestler, 1967).
} 
En el contexto del COVID-19, se ha transmutado un mandato impostergable: sólo un cambio radical en la comprensión de las interrelaciones humanas y las relaciones del ser humano con el ecosistema global, será capaz de frenar la pesadilla a la que nos ha llevado la irracionalidad capitalista: la destrucción del ser humano en el enfrentamiento con la naturaleza (OpenDEMOCRACY, 2020).

Este trabajo explora la genética del pensamiento complejo y del caos (proceso en el cual la potencia de la autoorganización y la autopoiesis resultan fundamentales) con la mira puesta en la propuesta emergente del bioaprendizaje; en coherencia con esta visión holística de la vida sobre el planeta y la necesidad de identificar opciones que permitan radicalizar la reflexión y comprensión respecto de la multiplicidad, interacciones y problemáticas en que se encuentra inmerso el ser humano. Una verdadera reconciliación del conocimiento humano con su fundamento biológico, el cual vale la pena explorar como punto de apoyo a partir del cual se vuelva a revolucionar el mundo.

Las siguientes líneas tienen por objetivo explorar con mayor detenimiento los orígenes y posterior desarrollo de la hipótesis Gaia, partiendo de pensadores que aportan a la cuestión desde sus reflexiones en torno a la autoorganización, interconectividad, complejidad, la física cuántica y el caos, hasta llegar a la autopoiesis y el bioaprendizaje.

Con este pequeño aleteo de mariposa, esperamos contribuir a dilucidar una salida posible, una mediación pedagógica, frente a la tragedia dantesca que se ha terminado por imponer con el episodio que representa la actual pandemia del COVID-19.

\section{¿Qué es la vida?}

En su libro ¿qué es la vida? (2005), Lynn Margulis junto a su hijo Dorion Sagan (hijo del reconocido astrofísico, Carl Sagan), se interrogan por la clave físico-biológica de la vida. Su objetivo, reconocer los fundamentos científicos con apoyo en los cuales fuera posible comprobar la tesis de que la biosfera constituye un organismo viviente.

Margulis y Sagan (2005) inician su búsqueda rememorando al físico austriaco Erwin Schrödinger (famoso por "El experimento del gato de Schrödinger" o "Paradoja de Schrödinger", 1935), quien en 1944 plantea al mundo entero la cuestión de ¿qué es la vida?

La respuesta que el propio Schrödinger dio en ese momento, era que la vida se caracterizaba por seguir un mismo patrón de reproducción, aquel que denominó, el del cristal aperiódico’.

Debido a la complejidad que representa exponer y comprender la conceptualización física-matemática del cristal aperiódico, Schrödinger (1983) propone la siguiente metáfora:

Si los cristales inorgánicos pueden ser entendidos como un papel pintado de pared, en el que el mismo dibujo se repite una y otra vez en períodos regulares, las fibras que componen el ADN, esencia de la célula viva, representan una obra maestra del bordado, por ejemplo, un tapiz de Rafael, que no presenta una repetición tediosa, sino un diseño elaborado, coherente y lleno de sentido, trazado por el gran maestro. (págs.19-20)

\footnotetext{
7. Según Schrödinger (1944), si los sólidos son cristales y en ese tanto son permanentes, el material hereditario no puede ser un cristal, ya que no se repite. Acuña entonces el término de cristal aperiódico, para referir a la naturaleza aperiódica del material genético, característica que permite codificar un número casi infinito de posibilidades con un número pequeño de átomos.
} 
A pesar que esta tesis es desechada, logra anticipar la importancia que tendrá en el desarrollo posterior de la ¿cuestión?, la interpretación de la vida como un patrón que se repite. Aunque Margulis y Sagan (2005) resultan insatisfechos con la tesis anterior, sobre todo por el excesivo reduccionismo de la respuesta, el desafío consiguió germinar en la próxima generación de científicos, encontrando un decidido apoyo en investigadores como la propia Lynn Margulis, Francisco Varela, Humberto Maturana, Fritjof Capra, Rupert Sheldrake, entre muchos otros.

Schrödinger había logrado triunfar, haciendo germinar la pregunta ¿qué es la vida? en la imaginación de sus colegas.

A pesar de la variedad de respuestas científicas que en el último siglo se han desarrollado para responder a la cuestión, gracias al trabajo de investigación, documentación y reflexión de Margulis y Sagan (2005), hoy se puede apreciar que en su gran mayoría, estas forman parte de un mismo esfuerzo conceptual: la apuesta por una visión holística de la vida, la que abiertamente buscaba superar las premisas del mecanicismo cartesiano.

Como bien señalan Margulis y Sagan (2005), si bien la comprensión mecánica de la vida impulsó de manera nunca antes vista la investigación científica, tanto como el conocimiento del universo entero, también significó la renuncia a una comprensión holística de la vida: aquella que se funda en los principios de cooperación, autopoiesis y transformación constante, los cuales interpreta como caracteres más importantes.

En su lugar, el mecanicismo redujo la vida a un conjunto de mecanismos físico-químicos que, unidos y sometidos a una serie de leyes universales, incluyendo la competencia como factor de evolución, permiten explicarla de una vez por todas.

Frente a esta forma reduccionista de entender la vida, los científicos y científicas que han apostado por el caos y la complejidad, han llevado adelante una importante dilucidación. La búsqueda de unos fundamentos gracias a los cuáles sea posible recuperar una comprensión holística del ser humano, capaz de superar la tendencia a imponer una organización jerárquica de la naturaleza e impulsando en su lugar una estructura de carácter rizomático (Deleuze y Guattari, 1966).

Desde el campo de filosofía, Deleuze y Guattari (1966; 1973) (reconocidos por su influencia en el pensamiento del siglo XX) empujaron la comprensión del caos, la fragmentación y la multiplicidad del mundo, introduciendo en el debate el concepto de rizoma, punto de apoyo para el enfrentamiento con la visión unitaria y lineal, jerárquica y ordenada de la realidad natural.

Por los principios de conexión y heterogeneidad, cualquier punto del rizoma puede ser conectado con cualquier otro, y debe serlo; por el principio de multiplicidad, sólo cuando lo múltiple es tratado efectivamente como multiplicidad, deja de tener relación con la unidad. (Deleuze y Guattari, 1966, págs.12-14)

Para estos autores, la vida puede ser entendida como un rizoma, en tanto la multiplicidad que impulsa permite explicar la increíble capacidad de combinación y recombinación de la naturaleza.

Los bulbos, los tubérculos, son rizomas. Pero hay plantas con raíz o raicilla que desde otros puntos de vista también pueden ser consideradas rizomorfas. Cabría, pues, preguntarse si la botánica, en su especificidad, no es enteramente rizomorfa. Hasta los animales lo son cuando van en manada, las ratas son rizomas. Las madrigueras lo son en todas sus funciones de hábitat, de provisión, de desplazamiento, de guarida y de ruptura. (Deleuze y Guattari, 1973, p.12) 
Si la evolución, como afirman Margulis y Sheldrake (1990), depende de la no competencia y la jerarquía, sino de la colaboración (sea que la explicación se encuentre en los campos morfogénicos o la combinación, acoplamiento y recombinación), los logros alcanzados en la búsqueda del carácter esencial de la vida no pueden ser más que calificados como un salto evolutivo en la comprensión que tiene el ser humano del mundo. Un verdadero caso de resonancia mórfica.

De acuerdo con Sheldrake (1991), bioquímico y biólogo británico, que toma las ideas de Carl Jung (1939) respecto de la sincronicidad y el inconsciente colectivo, existe una memoria mórfica en cada especie de seres vivos. Esta memoria registra las transformaciones y cambios evolutivos de la especie en el pasado. A su vez, existe una resonancia mórfica, la cual, por interacciones invisibles, no causales, no locales, en el tiempo y el espacio, influencia a cada individuo de la especie en su desarrollo orgánico y psíquico, al mismo tiempo que, permite a estos ejercer una influencia mórfica hacia el futuro, si logran superar las problemáticas que ponen en riesgos su supervivencia (tal como lo han hecho el cambio climático y el COVID-19, en nuestra era).

\section{La vida como autopoiesis}

Desde Schrödinger hasta Prigogine (Premio Nobel de Química de 1977, por sus investigaciones y conceptualización de las estructuras disipativas), se verifica un avance constante en la comprensión del orden no jerárquico de la naturaleza, siendo que cada uno de los autores y autoras que aportan en esta tarea, realizan en su momento un aporte fundamental que impulsa al siguiente, y así sucesivamente, hasta que se ha llegado a alcanzar la noción de autoorganización y de esta, el salto a la autopoiesis.

Entre los principales científicos y aportes, encontramos los siguientes:

- Richard Buckminster Fuller: arquitecto y activista medioambiental. Se vio motivado toda su vida ante la pregunta ¿tiene la humanidad una posibilidad de sobrevivir final y exitosamente en el planeta Tierra y, si es así, ¿cómo? Por ello, introduce el término ¿sinergética? (Fuller, 1982), gracias al cual remite a las propiedades (emergentes) de un conjunto, que no pueden ser explicadas a partir de sus elementos constitutivos;

- Arthur Koestler: alude a la holarquía para referir a la ausencia de jerarquía y control en la organización de la naturaleza, la cual se cimenta sobre la existencia de patrones o fractales en los elementos que forman parte de la cadena que conforma la vida sobre la Tierra.

- Ilya Prigogine: quien con sus investigaciones sobre las estructuras disipativas, llega a la conclusión de que no existe tal cosa como el desorden, sino un orden libre o autoorganización del caos (Briggs y Peat, 1999).

Aunque la vida no se limita a la autoorganización, este último concepto se encuentra en el corazón de aquella noción que Margulis (2005) identifica como la mejor forma de caracterizar la vida: la autopoiesis.

Por otro lado, Margulis y Sagan (2005) encuentran una gran similaridad entre los conceptos de autoorganización y autopoiesis, otros como Francisco Varela ${ }^{8}$ hacen notar una distancia cualitativa entre ambos, pudiéndose afirmar que el primero hace referencia a un carácter particular del segundo, el cual podría ser calificado como fenómeno. Fenómeno cuya distinción fundamental tiene que ver con la "capacidad" de generar una identidad única, con propiedades emergentes radicalmente novedosas y que no son reducibles a sus componentes, sino, que "son el resultado de la cooperación entre seres vivos, gracias a un continuo proceso de sinergia autoinducida de combinación, acoplamiento y recombinación" (Margulis y Sagan, 2005, p.18).

8. Biólogo chileno, investigador de las neurociencias y ciencias cognitivas, quien junto Humberto Maturana introdujo el concepto de autopoiesis en la biología.

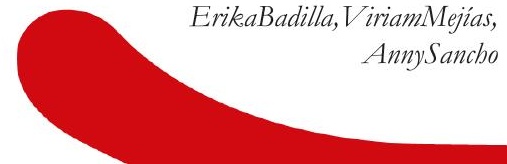


De acuerdo con Capra ${ }^{9}$ (1998), cuando Maturana ${ }^{10}$ y Varela (1972; 1974) acuñan el término autopoiesis para designar el carácter principal de la vida en general, aluden a una especie del género de la autoorganización, siendo tan así que, de la propia etimología del concepto se desprende una alusión a la capacidad de los seres vivos para auto crearse o autoorganizarse.

El propio Varela (2000), cuando relata el camino recorrido de antes y después que, junto con Maturana (1972), acuñan el término, hace notar que la organización autopoiética depende de la existencia de un mecanismo de separación parcial entre el ser vivo y su medio, el cual posibilite el surgimiento de una identidad propia, la interconexión interpretativa con el exterior, al mismo tiempo que la reproducción con variación estructural pero, con la conservación de la identidad.

Es evidente que el modelo en el cual se inspira la autopoiesis no es otro que la célula, forma de vida que según Margulis y Sagan (2005), constituye el patrón a partir del cual se desarrolla, en términos estructurales y evolutivos, el resto de la vida sobre el planeta Tierra: agrupaciones de células, organismo pluricelulares, comunidades de organismos, ecosistemas y la propia biosfera.

A pesar de lo anterior, Maturana y Varela (1972) incorporan otros patrones en su teoría: aquel según el cual la vida es una organización en red. Varela (1974) explicita que la autopoiesis se funda en la lógica celular básica: organización sistémica emergente, capaz de la automantención y autorregulación, con una barrera que determina el sí-mismo y no-sí-mismo; Assman (2002), que se interesa más que en un patrón básico, en la comprensión de los patrones dinámicos como forma en que un sistema se enfrenta a las exigencias de la supervivencia.

Llama la atención en este punto, el hecho de que todos los autores citados, si bien reconocen los avances y conocimientos que ha generado el mecanicismo, y con este, la mirada fisio-biológica de la vida, no dejan de mencionar la necesidad de aceptar que la vida va más allá de la materia y sus elementos fundamentales, alcanzando propiedades como la autoorganización, autopoiesis o la existencia de patrones de organización que son inexplicables, si se parte de la tradicional ciencia positivista.

Es en este punto que nos encontramos con la idea de estructuras disipativas, como concepto fundamental en la elucidación llevada adelante por los autores y autoras mencionadas.

Margulis y Sagan (2005), remiten nuevamente al premio nobel -Illya Progogine- señalando que la existencia de estructuras disipativas permite vislumbrar una relación entre la segunda ley de la termodinámica (los sistemas físicos tienden a disipar calor en su entorno) y la base de una acción aparentemente intencional, según la cual los sistemas retienen y transforman energía (combinan, acoplan y recombinan) para automantenerse, reintegrando y al medio aquellas formas menos útiles de energía. Para ambos autores, la vida se asemeja a corrientes de orden que tienden a mantener su forma, patrón o identidad.

Bajo esta concepción, la entropía que maximizan estas corrientes de orden, asimilables estas últimas con sistemas ordenados y disipativos, debe ser entendida no como mero desorden, sino como la generación progresiva de sistemas alejados del equilibrio. Es decir, que sólo pueden existir en conjunción con su entorno y que responden a principios distintos, como lo son los de no linealidad, fluctuación, bifurcación y autoorganización: esto es, con sistemas complejos.

\footnotetext{
9. Físico austriaco que ha dedicado su vida a reflexionar sobre las consecuencias filosóficas y sociales de la ciencia moderna.

10. Destacado biólogo y filósofo chileno, quien afirma que "los seres vivos somos sistemas autopoiéticos moleculares, o sea, sistemas moleculares que nos producimos a nosotros mismos, y la realización de esa producción de sí mismo como sistemas moleculares constituye el vivir" (Pais, 2019).
} 
O lo que es lo mismo, sistemas complejos cuyos caracteres, coinciden, precisamente, con aquellos otros que los autores y autoras mencionadas refrendan para una visión rizomática, biopedagógica de la vida.

Desde el punto de vista de Margulis y Sagan (2005), es esta relación entre el orden y la entropía, entre la diversidad de seres vivos y el medio ambiente, la que se encuentra detrás del exitoso proceso evolutivo que podemos apreciar en el planeta.

No es la competencia entre los seres vivos la que explica la evolución, sino la cooperación, la fusión entre seres que han alcanzado la estabilidad, en sistemas o nuevas unidades complejas de vida. Dicha estabilidad o adaptación al entorno, con fines de supervivencia y autopoiesis, puede ser entendido como un proceso de aprendizaje de los seres vivos en el entorno o sistema complejo del cual forman parte.

Como ya veremos, esta es una de las tesis fundamentales que proponen Assman (2002) y Varela (1974), respecto del bioaprendizaje.

\section{Propiedad emergente de la autopoiesis, el bioaprendizaje}

Como ya hemos adelantado, la autoorganización es explicada por Varela (1997) como la forma de organización de lo local, que no se funde en la existencia de reglas o elementos centrales de coordinación o una intervención externa, sino en altos niveles de cooperación (interacción) y coherencia entre los elementos, gracias a los cuales emergen nuevas propiedades para el conjunto.

Si la supervivencia de todo ser vivo depende de que este logre consolidar un nicho vital, y este se encuentra constituido por el organismo más su entorno (teoría del sistema unificado organismo/entorno), el pensamiento y la mente, no se puede más que comprender como encarnada. Esto es como una unidad con la corporalidad de ese organismo y la relación intrínseca entre este y su entorno.

En atención a la premisa de los sistemas unitarios de conocimiento (Varela, 2000; Varela et al., 1997), esta corporeidad del aprendizaje puede comprenderse gracias al término enacción, el cual refiere a esa cognición encarnada que constituye una interacción constante (acción) entre el organismo y el mundo, un desenvolvimiento creativo del sujeto cognoscente en atención a las particulares circunstancias en las cuales se encuentra inmerso.

En atención a la posición que asume todo organismo cognoscente frente a esta unidad mente-cuerpo-medio ambiente, Varela considera indispensable afirmar la existencia de una memoria biológica y social, producto de la interacción histórica de los elementos o subsistemas.

En coherencia con esta posición, Assmann ${ }^{11}$ afirma que la vida no es otra cosa que un aprendizaje constante. Dicha afirmación la funda este teólogo señalando que una de esas propiedades emergentes de la vida es el bioaprendizaje, el cual se funda en la inexistencia de una separación entre el proceso de vida y el proceso de aprendizaje. "Surge de esta forma una íntima conexión entre la comprensión de la vida como proceso biológico, y el aprendizaje como expresión del conocimiento implicado en dicho proceso" (Assman, 2002, p.39).

Este punto de vista también es compartido por Capra (1998), quien señala que el aprendizaje, la memoria y la toma de decisiones "son una consecuencia necesaria e inevitable de una cierta complejidad, que empieza mucho antes de que los organismos desarrollen cerebros y sistemas nerviosos superiores siendo que se presentan de igual forma en sistemas sociales y ecosistemas"(p.186).

11. Teólogo, católico y brasileño, uno de los principales impulsores de la Teología de la liberación. 
Vale agregar que, en Capra, la memoria resulta indispensable para el aprendizaje y la adaptación, encontrándose por ello presente no sólo en el cerebro humano, sino en prácticamente todos los sistemas y órganos del cuerpo, así como en todas las especies de seres vivos sobre el planeta. Así, se nos hace presente la afirmación hecha por Varela (2000) y Assman (2002), en el sentido de que la información, el conocimiento y el aprendizaje no tienen que ver con sistemas cerrados, estructuras a partir de un determinado orden interno, sino, que tiene que ver con las relaciones que se establecen entre el símismo y el no-sí-mismo, entre el individuo y su contexto o medio ambiente.

Si tomamos como referencia lo dicho por Margulis y Sagan (2005), lo anterior significa que el aprendizaje no sólo tiene que ver con el medio ambiente, como elemento indispensable para comprender los alcances y naturaleza del conocimiento, sino que al mismo tiempo y, sobre todo, implica que el ser humano tiene la tarea de reconocerse como parte (holon) de un todo sin el cual su existencia se encuentra condenada a terminar. (2002), afirma que la vida no es otra cosa que un aprendizaje constante. Dicha afirmación la funda este teólogo señalando que una de esas propiedades emergentes de la vida es el bioaprendizaje, el cual se funda en la inexistencia de una separación entre el proceso de vida y el proceso de aprendizaje. "Surge de esta forma una íntima conexión entre la comprensión de la vida como proceso biológico, y el aprendizaje como expresión del conocimiento implicado en dicho proceso" (Assman, 2002, p.39).

Este punto de vista también es compartido por Capra (1998), quien señala que el aprendizaje, la memoria y la toma de decisiones "son una consecuencia necesaria e inevitable de una cierta complejidad, que empieza mucho antes de que los organismos desarrollen cerebros y sistemas nerviosos superiores siendo que se presentan de igual forma en sistemas sociales y ecosistemas"(p.186).

Vale agregar que, en Capra, la memoria resulta indispensable para el aprendizaje y la adaptación, encontrándose por ello presente no sólo en el cerebro humano, sino en prácticamente todos los sistemas y órganos del cuerpo, así como en todas las especies de seres vivos sobre el planeta. Así, se nos hace presente la afirmación hecha por Varela (2000) y Assman (2002), en el sentido de que la información, el conocimiento y el aprendizaje no tienen que ver con sistemas cerrados, estructuras a partir de un determinado orden interno, sino, que tiene que ver con las relaciones que se establecen entre el símismo y el no-sí-mismo, entre el individuo y su contexto o medio ambiente.

Si tomamos como referencia lo dicho por Margulis y Sagan (2005), lo anterior significa que el aprendizaje no sólo tiene que ver con el medio ambiente, como elemento indispensable para comprender los alcances y naturaleza del conocimiento, sino que al mismo tiempo y, sobre todo, implica que el ser humano tiene la tarea de reconocerse como parte (holon) de un todo sin el cual su existencia se encuentra condenada a terminar.

\section{Hipótesis o conclusión}

Pedagógica y epistemológicamente hablando, frente a la teoría de los sistemas como unidad entre organismo y entorno, no es posible afirmar algo parecido a una transferencia de conocimiento, entre el ser humano y el entorno que percibe. Por el contrario, de acuerdo con esta tesis

El conocimiento es una de las formas de existencia del sistema unificado, complejo, al punto de que todo nuevo conocimiento no hace referencia a otra cosa que a modificaciones o transformaciones que se dan en la estructura misma del sistema. (Assman, 2002, p.36)

El conocimiento, a diferencia de lo que plantea el método científico (en su sentido mecanicista), no tiene que ver con unos supuestos poderes de percepción del ser humano, con la potencia de sus sentidos, sino con los alcances del sistema, así como a su continua autoorganización y autopoiesis.

El conocimiento es bajo esta lógica rizomática, el producto, una cualidad emergente, de la configuración que ha llegado a adquirir el sistema. 
Con lo que nos encontramos, bajo esta tesitura, no es con sentidos humanos que captan el entorno y le atribuyen sentido, sino con órganos que nos permiten crear relaciones entre el organismo y su entorno. Relaciones indispensables para la supervivencia y la actividad, instrumentos que le permiten al ser vivo comprobar aquellas hipótesis que respecto del entorno ya posee.

Como conclusión, este conocimiento entre relación organismo-sistema, en forma coherente Assman y otros autores adscritos a la tesis del bioaprendizaje, sostienen que toda percepción repercute de una u otra forma en la totalidad del sistema, tanto en el organismo como en el entorno.

Este último señalamiento coincide plenamente con lo dicho por Sheldrake, en el sentido de que los campos morfogénicos no son inamovibles, sino, que responden a una dinámica de continua transformación, adaptación, evolución, dinámicos, autoorganización, subsecuencia, acumulación y variación.

Se nos presenta así, como parte de este ejercicio de pensamiento rizomático, que la autoorganización puede ser catalogada con un bulbo conceptual, en el que la naturaleza entera, no solamente los seres vivos (si es que se puede afirmar, con seriedad, que el universo no está lleno de vida), sino también toda la materia que se nos aparece inerte bajo la visión mecanicista, tiende continuamente a organizarse, a crear patrones cada vez más complejos, lejos de la estabilidad que supone la termodinámica tradicional.

Desde este punto de mira, aprender no se traduce en acumular conocimientos, sino que el verbo más adecuado para comprender la aprehensión es el de albergar: estados inestables de unidad organismo-sistema, que le permiten al cerebro-mente proponer relaciones flexibles, creativas, rápidas con el entorno; relaciones que modifican el campo morfogénico, el proceso de autoorganización del sistema, incluida la morfogénica neuronal.

Margulis y Sagan (2005), Sheldrake (1990) y Varela (2000) sostienen que la cognición o aprendizaje, representa una propiedad emergente de toda red biológica o al interior de un sistema biológico. Conceptualización que, de forma coincidente, empata con la afirmación, hecha por Capra (1998), de la red como patrón de la organización biológica.

Así las cosas, si bien la autopoiesis constituye una noción atribuida a Maturana y Varela (1972) y Margulis (2005) demuestran que se trata en realidad de una conceptualización que recupera una visión holística de la vida, sistematizando una serie de ideas evolutivas, todas las cuales coinciden en afirmar la unidad inextricable entre el ser humano y la vida en el planeta Tierra.

En momentos en que científicos de todo el planeta afirman que problemáticas como el cambio climático y el COVID-19 son resultado de la actual forma de vida humana, de la explotación, despojo y desprecio de la vida sobre el planeta, y, que, de no darse una transformación radical en nuestro pensamiento, los efectos serán catastróficos. La hipótesis Gaia adquiere una relevancia quizás insospechada, teniendo en cuenta que en algún momento fue calificada como locura y herejía.

Las ideas expuestas en las líneas anteriores fueron desarrolladas y demostradas por científicos y científicas de todo el mundo, empeñadas en la tarea de revolucionar la comprensión que como seres humanos tenemos de la vida.

No se trata de meras especulaciones, se trata de explicaciones fundadas en datos científicos. No es posible continuar impasibles frente a esta realidad, replicando formas mecanicistas y reduccionistas de entender la vida, cuando la humanidad ya dispone de un conocimiento novedoso y creativo que le permite transformar completamente, no solo la sociedad, sino también, detener la destrucción de este amado planeta llamado Gaia.

Si Schrödinger lanzó el reto de comprender la esencia de la vida, quizás sea hora de asumir el que Margulis y Sagan (2005) y Lovelock (1983) expusieron hace cuatro décadas: el ser humano es solo un componente de 
la biosfera, si esta desaparece, nuestra especie también lo hará; si el objetivo de todo ser viviente es la supervivencia, nuestra obligación no es otra que asegurar la supervivencia de todas las especies sobre el planeta.

\section{Referencias Bibliográficas}

Assman, H. (2002). Placer y ternura en la educación: hacia una sociedad aprendiente. Madrid, España.: Narcea.

Blasco, J. (23 de mayo de 2017). El modelo original de la ciudad-jardín (Garden City) [Mensaje en un blog]. Recuperado de: http:/ / urban-networks.blogspot.cl/

Briggs, J., y Peat, D. (1999). Las siete leyes del caos. Barcelona, España.: Editorial Grijalbo.

Capra, F. (1998). La trama de la vida. (D. Sempau, Trad.). Barcelona, España: Anagrama Barcelona.

Deleuze, G., y Guattari, F. (1966). Rizoma: Introducción. (C. Casillas y V. Navarro, Trad.). México D.F: Ediciones Coyoacán.

Deleuze, G., y Guattari, F. (1973). El antiedipo. (1ª Ed., F. Monge, Trad.). Barcelona, España: Barral Editores.

Fuller, R. B. (1982). Synergetics: explorations in the geometry of thinking. United States: Macmillan Pub Co

Koestler, A. (1967). The Ghost in the Machine. London, England: Hutchinson.

Margulis, L., y Sagan, D. (2005). ¿Qué es la vida? (2 Ed.). Barcelona, España: Tusquets Editores.

Maturana, H., y Varela, F. (1972). De máquinas y seres vivos. Santiago, Chile: Editorial Universitaria.

Lovelock, J. E. (1983). Gaia: una nueva visión de la vida sobre la tierra. Madrid, España: Hermann Blume.

O’callaghan, C. (2020). Salud planetaria y COV ID-19: la degradación ambiental como el origen de la pandemia actual [Mensaje en un blog]. Recuperado de https://bit.ly/3kR6JLz.

OpenDEMOCRACY. (2020). COV TD-19: ¿última oportunidad para reevaluar nuestros valores? Recuperado de https://bit.ly/3bYrUr2

Pais, A. (23 de enero de 2019). La autopoiesis de Humberto Maturana, la definición de vida del biólogo chileno que hizo reflexionar hasta al dalái lama. BBC News Mundo. Recuperado de bttps://bbc. in/3k.QYiTq

Schrödinger, E. (1983). ¿Qué es la vida?. Barcelona, España: Tusquets Editores.

Sheldrake, R. (1990). La presencia del pasado: Resonancia mórfica y hábitos de la naturaleza. Barcelona, España: Kairós. Recuperado de bttps://bit.ly/3kNxmRP

Sheldrake, R. (1991). Resonancia mórfica. En S. Grof, Sabiduría antigua y ciencia moderna (147-164). Santiago, Chile: Cuatro Vientos.

Varela, F. (2000). El fenómeno de la vida. Santiago, Chile: Dolmen Ediciones.

Varela, F. G., Maturana, H. R., y Uribe, R. (1974). Autopoiesis: the organization of living systems, its characterization and a model. Biosystems, 5(4), 187-196. 
Varela, F., Thompson, E., y Rosch, E. (1997). De cuerpo presente. Las ciencias cognitivas y la experiencia humana. (2ª Ed., C. Gardini, Trad.). Barcelona, España: Gedisa. 